\title{
Photodesorption of ices - Releasing organic precursors into the gas phase
}

\author{
Karin I. Öberg ${ }^{1}$, Ewine F. van Dishoeck ${ }^{2}$, and Harold Linnartz ${ }^{1}$ \\ ${ }^{1}$ Raymond and Beverly Sackler Laboratory for Astrophysics \\ Leiden Observatory, Leiden University \\ P.O. Box 9513, NL-2300 RA Leiden, the Netherlands \\ email: oberg@strw.leidenuniv.nl, linnartz@strw.leidenuniv.nl \\ ${ }^{2}$ Leiden Observatory, Leiden University \\ P.O. Box 9513, NL-2300 RA Leiden, the Netherlands \\ email: ewine@strw.leidenuniv.nl
}

\begin{abstract}
A long-standing problem in interstellar chemistry is how molecules can be maintained in the gas phase at the extremely low temperatures in space. Photodesorption has been suggested to explain the observed cold gas in cloud cores and disk mid-planes. We are studying the UV photodesorption of ices experimentally under ultra high vacuum and at astrochemically relevant temperatures $(15-27 \mathrm{~K})$ using a hydrogen discharge lamp $(7-10.5 \mathrm{eV})$. The ice desorption during irradiation is monitored using reflection absorption infrared spectroscopy and the desorbed species using mass spectrometry. We find that both the UV photodesorption rates and mechanisms are highly molecule specific. CO photodesorbs without dissocation from the surface layer of the ice. $\mathrm{N}_{2}$, which lacks dipole allowed electronic transitions in the range of the lamp, does not photodesorb. $\mathrm{CO}_{2}$ desorbs through dissociation and subsequent recombination from the top few layers of the ice. At low temperatures $(15-18 \mathrm{~K})$ the derived photodesorption rates are $\sim 10^{-3}$ for $\mathrm{CO}$ and $\mathrm{CO}_{2}$ and $<2 \times 10^{-4}$ for $\mathrm{N}_{2}$ ice per incident photon.
\end{abstract}

Keywords. Astrochemistry, molecular data, molecular processes, methods: laboratory

\section{Introduction}

$\mathrm{CO}$ and $\mathrm{CO}_{2}$ ice are among the most abundant species in star forming regions, after $\mathrm{H}_{2}$, and, e.g., Tielens \& Charnley (1997) show that they form the building blocks of a complex organic chemistry once desorbed into the gas phase. A long-standing problem is how these molecules can be maintained in the gas phase at the low temperatures found in star forming regions, where all molecules other than $\mathrm{H}_{2}$ should stick on dust grains on timescales shorter than the cloud and outer disk lifetimes. Yet lines of gaseous CO are detected towards dark clouds (e.g., Bergin et al. 2002). Similarly, Piétu et al. (2007) observed abundant gas-phase $\mathrm{CO}$, which cannot be explained by thermal equilibrium chemistry, in the cold parts of protoplanetary disks. Very recently Sakai et al. (2008) detected cold $\mathrm{HCO}_{2}^{+}$(tracing $\mathrm{CO}_{2}$ ), toward a protostar as well. Photodesorption of ices has been suggested to explain these kinds of observations and Öberg et al. (2007) showed that CO photodesorption is an efficient process with a rate of $3( \pm 1) \times 10^{-3}$ photon $^{-1}$. We also reported that $\mathrm{N}_{2}$ does not photodesorb. In this study we extend the $\mathrm{CO}$ and $\mathrm{N}_{2}$ photodesorption study to include different ice temperatures and morphologies. We also present the first results on the photodesorption rate of $\mathrm{CO}_{2}$ and its dependences on ice thickness, UV flux, irradiation time and total UV dose. 


\section{Experimental and Data Analysis}

In the photodesorption experiments, thin ices of 2 to 100 monolayers (ML) are grown at $15-27 \mathrm{~K}$ on a gold substrate under ultra-high vacuum conditions $\left(P \sim 10^{-10} \mathrm{mbar}\right)$. The ice films are subsequently irradiated with UV light from a broadband hydrogen microwave discharge lamp, which covers 120-170 nm (7-10.5 eV). The setup allows simultaneous detection of molecules in the gas phase by quadropole mass spectrometry (QMS) and in the ice by reflection absorption infrared spectroscopy (RAIRS) using a Fourier transform infrared spectrometer. The ice UV destruction rate is determined by RAIR spectroscopy of the ice during irradiation as described by Öberg et al. (2007). In general we define the photodesorption rate as the destruction rate of the original ice minus the formation rate of other ice species. $\mathrm{N}_{2}$ has no permanent dipole moment and is not detected with RAIRS. Instead the photodesorption upper limit is constrained using only QMS measurements.

\section{Results and Discussion}

In the extended investigation of $\mathrm{CO}$ ice photodesorption, we find that the desorption rate decreases with increasing temperature such that it is almost a factor of 3 lower at $27 \mathrm{~K}$ compared to at $15 \mathrm{~K}$. When the ice is deposited at $27 \mathrm{~K}$ and then cooled down to $16 \mathrm{~K}$ before irradiation the rate is a factor of 3 lower as well, showing that the $\mathrm{CO}$ ice photodesorption depends on the ice structure (as modified by annealing) rather than temperature. An additional experiment with a CO: $\mathrm{N}_{2}=4: 4 \mathrm{ML}$ mixed ice indicates a slight $\mathrm{N}_{2}$ co-desorption with $\mathrm{CO}$ at a rate of $\sim 3 \times 10^{-4}$ photon $^{-1}$. This $\mathrm{N}_{2}$ co-desorption may provide the means of releasing $\mathrm{N}_{2}$ ice into the gas phase non-thermally, despite its low UV absorption cross section.

In the new $\mathrm{CO}_{2}$ ice experiments, the derived $\mathrm{CO}_{2}$ photodesorption rate has no measurable dependence on lamp flux, irradiation time or total photon dose at $18 \mathrm{~K}$. There is also no measurable isotope dependence between ${ }^{13} \mathrm{C}^{18} \mathrm{O}_{2}$ and ${ }^{13} \mathrm{C}^{16} \mathrm{O}_{2}$. The $\mathrm{CO}_{2}$ photodesorption rate at $18 \mathrm{~K}$ is however dependent on ice thickness up to several monolayers; the rate increases from $\sim 0.7$ to $1.8 \times 10^{-3}$ photon $^{-1}$ when the ice is grown from 2 to $10 \mathrm{ML}$. Beyond $10 \mathrm{ML}$, the rate is thickness-independent, indicating that only the top 10 ML can photodesorb. This is in contrast with the surface layer desorption of CO ice. The difference can be understood from the different photodesorption mechanisms of CO and $\mathrm{CO}_{2}$; $\mathrm{CO}$ photodesorption is predicted to occur on surfaces only (Takahashi \& van Hemert, in prep.), while the dissociation of $\mathrm{CO}_{2}$ prior to desorption produces energetic fragments, which can penetrate several ice layers. When including photodesorption of different ices in astrophysical models these different thickness dependences need to be taken into account to accurately predict the photodesorption efficiencies.

In general this study shows that at least two ices are efficiently photodesorbed and we recommend that photodesorption be included in models where non-thermal desorption is expected to affect the model outcome.

\section{References}

Bergin, E. A., Alves, J., Huard, T., \& Lada, C. J. 2002, ApJ (Letters), 570, L101

Öberg, K. I., Fuchs, G. W., Awad, Z., et al. 2007, ApJ (Letters), 662, L23

Piétu, V., Dutrey, A., \& Guilloteau, S. 2007, A\& A, 467, 163

Sakai, N., Sakai, T., Aikawa, Y., \& Yamamoto, S., 2008, ApJ (Letters), 675, L89

Tielens, A. G. G. M. \& Charnley, S. B. 1997, Origins of Life and Evolution of the Biosphere, 27,23 